\title{
Breaking up Simplicial Homology and Subadditivity of Syzygies*
}

\author{
Sara Faridi $\quad$ Mayada Shahada ${ }^{\ddagger}$
}

January 27,2022

\begin{abstract}
We consider the following question: if a simplicial complex $\Gamma$ has $d$-homology, then does the corresponding $d$-cycle always induce cycles of smaller dimension that are not boundaries? We provide an answer to this question in a fixed dimension. We use the breaking of homology to show the subadditivity property for the maximal degrees of syzygies of monomial ideals in a fixed homological degree.
\end{abstract}

\section{Introduction}

The motivation for this paper is the subadditivity property for the maximal degrees of syzygies of monomial ideals in polynomial rings. Let $I$ be a homogeneous ideal in the polynomials ring $S=k\left[x_{1}, \ldots, x_{n}\right]$ over a field $k$. Let $t_{a}$ denoted the maximum value of $j$ such that the graded Betti number $\beta_{a, j}(S / I) \neq 0$. The ideal $I$ satisfies the subadditivity property on the maximal degrees of its syzygies if

$$
t_{a+b} \leqslant t_{a}+t_{b}
$$

where $a+b$ is not more than the projective dimension of the ideal.

The inequality in (11) arises most naturally in the context of (Castelnuovo-Mumford) regularity, which, for the ideal $I$, can be described as the maximum value of $t_{a}-a$, for all positive integers $a$. It has been shown to fail in general by Avramov, Conca and Iyengar [2], even if one restricts to Cohen-Macaulay or even Gorenstein settings (see [22] for examples and for a general survey on the topic). However, many special cases are known: certain algebras with codimension $\leqslant 1$ (Eisenbud, Huneke and Ulrich [11]), certain classes of Koszul rings (Avramov, Conca and Iyengar [2]), certain homological degrees for Gorenstein algebras (El Khoury and Srinivasan [12]), among others.

Avramov, Conca and Iyengar [2] conjectured that the subadditivity property holds for Kozul rings and for all monomial ideals (it is also open for toric ideals [22]). In the case of monomial ideals, there are special cases for which (1) has been verified: when $a=1$ (Herzog and Srinivasan [19]), when $a=1,2,3$ and $I$ is generated in degree 2 (Fernández-Ramos and Gimenez [15], Abedelfatah

*Subject Classification 13A15, 13D02, 13F55, 05E40, 05E45

† Keywords: monomial ideals, subadditivity, simplicial homology

Department of Mathematics and Statistics, Dalhousie University, Halifax, Canada, faridi@ dal.ca

${ }^{\S}$ Department of Mathematics, University of Bahrain, Sakheer, Kingdom of Bahrain, mshahada@uob.edu.bh 
and Nevo [3]), Cohen-Macaulay ideals generated by monomials of degree 2 when the base field has characteristic 0 [2], facet ideals of simplicial forests (Faridi [13]), ideals whose Betti diagram has a special "shape" (Bigdeli and Herzog [5]), several classes of edge ideals of graphs and path ideals of rooted trees (Jayanthan and Kumar [20]), and for $a$ where the Stanley-Reisner complex of $I$ has dimension bounded by $t_{a}-a$ (Abedelfatah [1]).

In the case of monomial ideals, the syzygies can be characterized as dimensions of homology modules of topological objects. This is one of the central themes of Stanley-Reisner Theory, connecting Commutative Algebra to Discrete Geometry and Topology. We refer the reader to the books [6, 24] for more details on these rich connections.

By viewing the subadditivity property as a geometric one, the inequality in (1) can be shown to follow from the following general type of question:

Does a topological object with d-homology break into sub-objects that have a-homology and $b$-homology, where $a$ and $b$ are related to $d$ ?

This approach was taken by the first author in [13], where the topological objects were atomic lattices (lcm lattices of monomial ideals); see Question 2.1 and Question 2.2 below. In this paper, using Hochster's formula (Equation (2)), we examine this problem from the point of view of the Stanley-Reisner complex, and we can provide a positive answer to the general question above for a fixed value of $d$. As a result we show that subadditivity holds in a fixed homological degree for all monomial ideals. The last section interprets the square-free results of the paper for general monomial ideals.

\section{Acknowledgements}

The authors are grateful to the referees and Kieran Bhaskara, whose comments improved the paper. The second author would like to acknowledge the support of Dalhousie University where she was a postdoc while this research was carried out. The research of the first author was supported by an NSERC Discovery Grant.

\section{Setup}

\subsection{The subadditivity property}

Throughout the paper, let $S=k\left[x_{1}, \ldots, x_{n}\right]$ be a polynomial ring over a field $k$. If $I$ is a graded ideal of $S$ with minimal free resolution

$$
0 \rightarrow \oplus_{j \in \mathbb{N}} S(-j)^{\beta_{p, j}} \rightarrow \oplus_{j \in \mathbb{N}} S(-j)^{\beta_{p-1, j}} \rightarrow \cdots \rightarrow \oplus_{j \in \mathbb{N}} S(-j)^{\beta_{1, j}} \rightarrow S,
$$

then for each $i$ and $j$, the $\operatorname{rank} \beta_{i, j}(S / I)$ of the free $S$-modules appearing above are called the graded Betti numbers of the $S$-module $S / I$.

If we let

$$
t_{a}=\max \left\{j: \beta_{a, j}(S / I) \neq 0\right\},
$$

a question is whether the $t_{a}$ satisfy the subadditivity property: $t_{a+b} \leqslant t_{a}+t_{b}$ ? 
The answer is known to be negative for a general homogeneous ideal [2], and unknown in the case of monomial ideals. For the case of monomial ideals, there are special cases that are known [19, 3, 15, 13, 5, 1].

In the case of monomial ideals, Betti numbers can be interpreted as the homology of objects in discrete topology: simplicial complexes, order complexes of lattices, etc.; see for example [24] for a survey of this approach. As a result, the subadditivity question can be viewed as a question of breaking up homology in these objects. This idea was explored in [13] by the first author, where the subadditivity problem was solved for facet ideals of simplicial forests using homology of lattices.

By a method called polarization [16] (see Section 5for the definition), one can reduce questions regarding Betti numbers of monomial ideals to the class of square-free monomial ideals.

If $u \subset[n]=\{1, \ldots, n\}$, then we define

$$
\mathbf{m}_{u}=\prod_{i \in u} x_{i}
$$

to be the square-free monomial with support $u$.

For our purposes it is useful to consider a finer grading of the Betti numbers by indexing the Betti numbers with monomials of the polynomial ring $S$. A multigraded Betti number of $S / I$ is of the form $\beta_{i, \mathbf{m}}(S / I)$ where $\mathbf{m}$ is a monomial in $S$ and

$$
\beta_{i, j}(S / I)=\sum_{u \subseteq[n] \text { and }|u|=j} \beta_{i, \mathbf{m}_{u}}(S / I) .
$$

\subsection{Simplicial Complexes}

A simplicial complex $\Gamma$ on a set $W$ is a set of subsets of $W$ with the property that if $F \in \Gamma$ then for every subset $G \subseteq F$ we have $G \in \Gamma$. Every element of $\Gamma$ is called a face, the maximal faces under inclusion are called facets, and a simplicial complex contained in $\Gamma$ is called a subcomplex of $\Gamma$. The set of all $v \in W$ such that $\{v\} \in \Gamma$ is called the vertex set of $\Gamma$, and is denoted by $V(\Gamma)$. The set of facets of $\Gamma$ is denoted by $\operatorname{Facets}(\Gamma)$. If $\operatorname{Facets}(\Gamma)=\left\{F_{1}, \ldots, F_{q}\right\}$, then we denote $\Gamma$ by

$$
\Gamma=\left\langle F_{1}, \ldots, F_{q}\right\rangle .
$$

If $A \subset V(\Gamma)$, then the induced subcomplex $\Gamma_{A}$ is defined as

$$
\Gamma_{A}=\{F \in \Gamma: F \subseteq A\} .
$$

The Alexander dual $\Gamma^{\vee}$ of $\Gamma$, if we set $F^{c}=V(\Gamma) \backslash F$, is defined as

$$
\Gamma^{\vee}=\left\{F \subseteq V(\Gamma): F^{c} \notin \Gamma\right\}=\{V(\Gamma) \backslash F: F \notin \Gamma\} .
$$

The link of a face $F$ of $\Gamma$ is

$$
\operatorname{lk}_{\Gamma}(F)=\{G \in \Gamma: F \cap G=\emptyset \text { and } F \cup G \in \Gamma\} .
$$

If $I$ is a square-free monomial ideal in $S$, it corresponds uniquely to a simplicial complex

$$
\mathcal{N}(I)=\left\{u \subset[n]: m_{u} \notin I\right\}
$$


called the Stanley-Reisner complex of $I$. Conversely, if $\Gamma$ is a simplicial complex whose vertices are labelled with $x_{1}, \ldots, x_{n}$, then one can associate to it its unique Stanley-Reisner ideal

$$
\mathcal{N}(\Gamma)=\left\{m_{u}: u \subset[n] \text { and } u \notin \Gamma\right\}
$$

The uniqueness of the Stanley-Reisner correspondence implies that

$$
\mathcal{N}(\Gamma)=I \Longleftrightarrow \mathcal{N}(I)=\Gamma \text {. }
$$

\subsection{The lcm lattice}

A lattice is a partially ordered set where every two elements have a greatest lower bound called their meet and a lowest upper bound called their join. A bounded lattice has an upper and a lower bound denoted by $\hat{1}$ and $\hat{0}$, respectively.

If $L$ is a lattice with $r$ elements, then the order complex of $L$ is the simplicial complex on $r$ vertices, where the elements of each chain in $L$ form a face.

If $I$ is a monomial ideal, then the $\mathbf{l c m}$ lattice of $I$, denoted by $\operatorname{LCM}(I)$, is a bounded lattice ordered by divisibility, whose elements are the generators of $I$ and their least common multiples, and the meet of two elements is their least common multiple.

Two elements of a lattice are called complements if their join is $\hat{1}$ and their meet is $\hat{0}$. If the lattice is $\operatorname{LCM}(I)$, then it was shown in [13] that two monomials in $\operatorname{LCM}(I)$ are complements if their gcd is not in $I$ and their $1 \mathrm{~cm}$ is the $1 \mathrm{~cm}$ of all the generators of $I$.

Gasharov, Peeva and Welker [17, 24] showed that multigraded Betti numbers of $S / I$ can be calculated from the homology of (the order complex of) the lattice $\operatorname{LCM}(I)$ : if $\mathbf{m}$ is a monomial in $L=\operatorname{LCM}(I)$, then

$$
\beta_{i, \mathbf{m}}(S / I)=\operatorname{dim}_{k} \tilde{H}_{i-2}\left((1, \mathbf{m})_{L} ; k\right)
$$

where $(1, \mathbf{m})_{L}$ refers to the subcomplex of the order complex consisting of all nontrivial monomials in $L$ strictly dividing $\mathbf{m}$.

On the other hand, in a 1977 paper, Baclawski [4] showed that if $L$ is a finite lattice whose proper part has nonzero homology, then every element of $L$ has a complement.

The following question was raised in [13] as a potential way to answer the subadditivity question.

Question 2.1. If $I$ is a square-free monomial ideal in variables $x_{1}, \ldots, x_{n}$, and $\beta_{i, n}(S / I) \neq 0, a, b>0$ and $i=a+b$, are there complements $\mathbf{m}$ and $\mathbf{m}^{\prime}$ in $\operatorname{LCM}(I)$ with $\beta_{a, \mathbf{m}}(S / I) \neq 0$ and $\beta_{b, \mathbf{m}^{\prime}}(S / I) \neq 0$ ?

Considering that it is enough to study the "top degree" Betti numbers (those of degree $n$, in this case) [9, 13], a positive answer to Question 2.1 will establish the subadditivity property for all monomial ideals, since

$$
t_{a}+t_{b} \geqslant \operatorname{deg}(\mathbf{m})+\operatorname{deg}\left(\mathbf{m}^{\prime}\right) \geqslant n=t_{i} .
$$

Question 2.1 can be written more generally as a question about the homology of the lcm lattice, or in fact, any finite lattice. 
Question 2.2. If $L=\operatorname{LCM}(I)$ and $\tilde{H}_{i-2}\left(\left(1, x_{1} \cdots x_{n}\right)_{L} ; k\right) \neq 0, a, b>0$ and $i=a+b$, are there complements $\mathbf{m}$ and $\mathbf{m}^{\prime}$ in $\operatorname{LCM}(I)$ with $\tilde{H}_{a-2}\left((1, \mathbf{m})_{L} ; k\right) \neq 0$ and $\tilde{H}_{b-2}\left(\left(1, \mathbf{m}^{\prime}\right)_{L} ; k\right) \neq 0$ ?

With the same idea, one could translate Question 2.1 into breaking up simplicial homology using Hochster's formula.

\subsubsection{Hochster's Formula}

Let $I=\left(\mathbf{m}_{1}, \ldots, \mathbf{m}_{q}\right)$ be a square-free monomial ideal in the polynomial ring $S=k\left[x_{1}, \ldots, x_{n}\right]$. Hochster's formula (see for example [18, Cor. 8.1.4 and Prop. 5.1.8]) states that if $I=\mathcal{N}(\Gamma)$ and $\mathbf{m}_{u}$ a monomial, then

$$
\beta_{i, \mathbf{m}_{u}}(S / I)=\operatorname{dim}_{k} \tilde{H}_{i-2}\left(\operatorname{lk}_{\Gamma^{\vee}}\left(u^{c}\right), k\right)=\operatorname{dim}_{k} \tilde{H}_{|u|-i-1}\left(\Gamma_{u}, k\right)
$$

where $u^{c}=[n] \backslash u$ is the set complement of $u$. We would now like to reinterpret Question 2.1 in the language of Hochster's formula. To begin with, since we are dealing with square-free monomials, we can consider a monomial $\mathbf{m}_{u}$ equivalent to the set $u$ and use intersections for gcd, unions for $1 \mathrm{~cm}$, and $\mathbf{m}_{u}^{c}$ for $u^{c}$.

Suppose

$$
\beta_{i, x_{1} \ldots x_{n}}(S / I)=\operatorname{dim}_{k} \tilde{H}_{i-2}\left(\mathrm{lk}_{\Gamma^{\vee}}(\emptyset), k\right)=\operatorname{dim}_{k} \tilde{H}_{i-2}\left(\Gamma^{\vee}, k\right) \neq 0
$$

and $i=a+b$ where $a, b>0$. We would like to know if there are complements $\mathbf{m}, \mathbf{m}^{\prime} \in \operatorname{LCM}(I)$ such that

$$
\beta_{a, \mathbf{m}}(S / I) \neq 0 \text { and } \beta_{b, \mathbf{m}^{\prime}}(S / I) \neq 0 .
$$

First observe that, $\Gamma^{\vee}=\left\langle\mathbf{m}_{1}^{c}, \ldots, \mathbf{m}_{q}^{c}\right\rangle$ (e.g. [18] or [14, Prop. 2.4]).

We have

$$
\begin{aligned}
\mathbf{m} \in \operatorname{LCM}(I) & \Longleftrightarrow \mathbf{m}=\mathbf{m}_{i_{1}} \cup \mathbf{m}_{i_{2}} \cup \cdots \cup \mathbf{m}_{i_{s}} \text { for some } 1 \leqslant i_{1}<i_{2}<\cdots<i_{s} \leqslant q \\
& \Longleftrightarrow \mathbf{m}^{c}=\mathbf{m}_{i_{1}}^{c} \cap \mathbf{m}_{i_{2}}^{c} \cap \cdots \cap \mathbf{m}_{i_{s}}^{c} \text { for some } 1 \leqslant i_{1}<i_{2}<\cdots<i_{s} \leqslant q \\
& \Longleftrightarrow \mathbf{m}^{c} \text { is the intersection of some facets of } \Gamma^{\vee} .
\end{aligned}
$$

Moreover, if $\mathbf{m}, \mathbf{m}^{\prime} \in \operatorname{LCM}(I)$, then

$$
\begin{aligned}
\mathbf{m}, \mathbf{m}^{\prime} \text { are complements } & \Longleftrightarrow \mathbf{m} \cup \mathbf{m}^{\prime}=[n] \text { and } \mathbf{m} \cap \mathbf{m}^{\prime} \notin I \\
& \Longleftrightarrow \mathbf{m}^{c} \cap \mathbf{m}^{c}=\emptyset \text { and } \mathbf{m} \cap \mathbf{m}^{\prime} \in \Gamma \\
& \Longleftrightarrow \mathbf{m}^{c} \cap \mathbf{m}^{c}=\emptyset \text { and }\left(\mathbf{m} \cap \mathbf{m}^{\prime}\right)^{c} \notin \Gamma^{\vee} \\
& \Longleftrightarrow \mathbf{m}^{c} \cap \mathbf{m}^{\prime c}=\emptyset \text { and } \mathbf{m}^{c} \cup \mathbf{m}^{c} \notin \Gamma^{\vee}
\end{aligned}
$$

So we are looking for subsets $A, B \subseteq[q]$ such that

1. $\mathbf{m}^{c}=\bigcap_{j \in A} \mathbf{m}_{j}^{c}$ and $\mathbf{m}^{c}=\bigcap_{j \in B} \mathbf{m}_{j}^{c}$

2. $\mathbf{m}^{c} \cap \mathbf{m}^{c}=\emptyset$ 
3. $\mathbf{m}^{c} \cup \mathbf{m}^{c} \notin \Gamma^{\vee}$

4. $\tilde{H}_{a-2}\left(\mathrm{lk}_{\Gamma^{\vee}}\left(\mathbf{m}^{c}\right), k\right) \neq 0$ and $\tilde{H}_{b-2}\left(\mathrm{lk}_{\Gamma^{\vee}}\left(\mathbf{m}^{c}\right), k\right) \neq 0$.

Now we can state Question 2.1 in the following form.

Question 2.3. If $\Gamma=\left\langle F_{1}, \ldots, F_{q}\right\rangle$ is a simplicial complex with $\tilde{H}_{i-2}(\Gamma, k) \neq 0$ and $i=a+b$ where $a, b>0$, can we find subsets $A, B \subseteq[q]$ such that

1. $F=\bigcap_{j \in A} F_{j}$ and $G=\bigcap_{j \in B} F_{j}$

2. $F \cap G=\emptyset$

3. $F \cup G \notin \Gamma$

4. $\tilde{H}_{a-2}\left(\mathrm{lk}_{\Gamma}(F), k\right) \neq 0$ and $\tilde{H}_{b-2}\left(\mathrm{lk}_{\Gamma}(G), k\right) \neq 0$ ?

Example 2.4. If $\mathcal{N}(I)^{\vee}=\Gamma=\langle x z u, x z v, x u v, y z u, y z v, y u v, x y\rangle$,

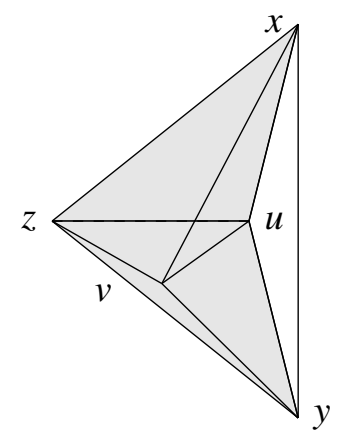

then $I=(x z, y z, x u, y u, x v, y v, z u v)$ has Betti table

$\begin{array}{rlllll} & 0 & 1 & 2 & 3 & 4 \\ \text { total : } & 1 & 7 & 11 & 6 & 1 \\ 0: & 1 & . & . & . & . \\ 1: & . & 6 & 9 & 5 & 1 \\ 2: & . & 1 & 2 & 1 & .\end{array}$

So $\beta_{i, \text { xyzuv }} \neq 0$ when $i=3$, 4, which corresponds to nonvanishing of homology of links of faces of $\Gamma$ in dimensions 1,2 . We consider each case separately:

1. $i=3, a=1, b=2$. Then $\tilde{H}_{1}(\Gamma, k) \neq 0$. Let $F=x y$ and $G=x u v \cap y u v=u v$, then $F \cap G=\emptyset$, $F \cup G=x y u v \notin \Gamma$, and

$$
\tilde{H}_{a-2}\left(\operatorname{lk}_{\Gamma}(F), k\right)=\tilde{H}_{-1}(\langle\emptyset\rangle, k) \neq 0 \text { and } \tilde{H}_{b-2}\left(\operatorname{lk}_{\Gamma}(G), k\right)=\tilde{H}_{0}(\langle x, y\rangle, k) \neq 0 \text {. }
$$


2. $i=4, a=1, b=3$. Then $\tilde{H}_{2}(\Gamma, k) \neq 0$. Let $F=y z u$ and $G=x z u \cap x u v \cap x z v \cap x y=x$, then $F \cap G=\emptyset, F \cup G=x y z u \notin \Gamma$, and

$$
\tilde{H}_{a-2}\left(\mathrm{lk}_{\Gamma}(F), k\right)=\tilde{H}_{-1}(\langle\emptyset\rangle, k) \neq 0 \text { and } \tilde{H}_{b-2}\left(\mathrm{lk}_{\Gamma}(G), k\right)=\tilde{H}_{1}(\langle z u, u v, z v, y\rangle, k) \neq 0 .
$$

3. $i=4, a=2, b=2$. Then $\tilde{H}_{2}(\Gamma, k) \neq 0$. Let $F=y z u \cap y u v=y u$ and $G=x z u \cap x z v=x z$, then $F \cap G=\emptyset, F \cup G=x y z u \notin \Gamma$, and

$$
\tilde{H}_{a-2}\left(\operatorname{lk}_{\Gamma}(F), k\right)=\tilde{H}_{0}(\langle z, v\rangle, k) \neq 0 \text { and } \tilde{H}_{b-2}\left(\mathrm{lk}_{\Gamma}(G), k\right)=\tilde{H}_{0}(\langle u, v\rangle, k) \neq 0 .
$$

A dual version of Question 2.3 can be stated as follows (see Corollary 3.6 for the justification).

Question 2.5. If $\Gamma$ is a simplicial complex on the vertex set $\left\{x_{1}, \ldots, x_{n}\right\}$, and $\tilde{H}_{i-2}(\Gamma, k) \neq 0$, and $n-i+1=a+b$, where $a$ and $b$ are positive integers, are there nonempty subsets $C, D \subseteq\left\{x_{1}, \ldots, x_{n}\right\}$ such that

1. $C \cup D=\left\{x_{1}, \ldots, x_{n}\right\}$

2. $C \cap D \in \Gamma$

3. $\tilde{H}_{|C|-a-1}\left(\Gamma_{C}, k\right) \neq 0$ and $\tilde{H}_{|D|-b-1}\left(\Gamma_{D}, k\right) \neq 0$ ?

Example 2.6. Let $\mathcal{N}(I)=\Gamma=\langle z w x, v w x, u v x, z u x, z u y, u v y, v w y, z w y\rangle$.

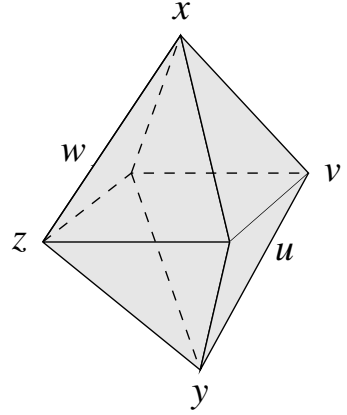

$\Gamma$

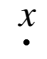

$\dot{y}$

$\Gamma_{C}$

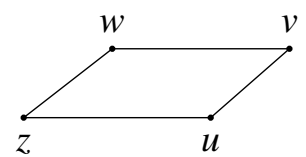

$\Gamma_{D}$

Then $I=(x y, z v, u w)$ has Betti table

$\begin{array}{rllll} & 0 & 1 & 2 & 3 \\ \text { total }: & 1 & 3 & 3 & 1 \\ 0: & 1 & . & . & . \\ 1: & . & 3 & . & . \\ 2: & . & . & 3 & . \\ 3: & . & . & . & 1\end{array}$


So $\beta_{3, \text { xyzuvw }}(S / I) \neq 0$ which corresponds to nonvanishing homology of $\Gamma$ in dimension 2 (i.e. $\left.\tilde{H}_{2}(\Gamma, k) \neq 0\right)$. Let $a=1$ and $b=2$. Choose $C=\{x, y\}$ and $D=\{z, u, v, w\}$. Then $C \cup D=$ $\{x, y, z, u, v, w\}, C \cap D=\emptyset \in \Gamma$ and

$$
\tilde{H}_{|C|-a-1}\left(\Gamma_{C}, k\right)=\tilde{H}_{0}(\langle x, y\rangle, k) \neq 0 \text { and } \tilde{H}_{|D|-b-1}\left(\Gamma_{D}, k\right)=\tilde{H}_{1}(\langle z u, u v, v w, z w\rangle, k) \neq 0 .
$$

A positive answer to either Question 2.3 or Question 2.5 would settle the subadditivity question for syzygies.

\section{Main results}

The following lemma is an easy exercise.

Lemma 3.1. $\Gamma$ simplicial complex and $A \in \Gamma$ and $B \in \mathrm{lk}_{\Gamma}(A)$, then

$$
\mathrm{lk}_{\mathrm{lk}_{\Gamma}(A)}(B)=\mathrm{lk}_{\Gamma}(A \cup B) .
$$

In a simplicial complex $\Gamma$ we say a $d$-cycle $\Sigma$ is supported on faces $F_{1}, \ldots, F_{q}$ if $\Sigma=a_{1} F_{1}+$ $\cdots+a_{q} F_{q}$ for nonzero scalars $a_{1}, \ldots, a_{q} \in k$. We say that $\Sigma$ is a face-minimal cycle or minimally supported on $F_{1}, \ldots, F_{q}$ if additionally no proper subset of $F_{1}, \ldots, F_{q}$ is the support of a $d$-cycle. If $\Sigma$ is supported on $F_{1} \ldots, F_{q}$, we call the simplicial complex $\left\langle F_{1}, \ldots, F_{q}\right\rangle$ the support complex of $\Sigma$.

Example 3.3 can guide the reader through the statement of the theorem below, a variation of which appears as Theorem 4.2 of [25].

Theorem 3.2. Let $k$ be a field, $\Gamma$ a d-dimensional simplicial complex, and

$$
\Sigma=a_{1} F_{1}+\cdots+a_{q} F_{q} \quad a_{1}, \ldots, a_{q} \in k
$$

a d-cycle in $\Gamma$ supported on $F_{1}, \ldots, F_{q}$ which is not a boundry, so that $\tilde{H}_{d}(\Gamma, k) \neq 0$. Suppose $A$ is a face of the support complex of $\Sigma$ such that for some $s \leqslant q$ we have

$$
A \subseteq F_{1} \cap \ldots \cap F_{s} \text {, and } A \nsubseteq F_{j} \text { if } j>s
$$

and $0 \leqslant|A| \leqslant d+1$. Then

1. there are $\epsilon_{i} \in\{ \pm 1\}$ for $i=1, \ldots$, s such that

$$
\Sigma_{A}=\epsilon_{1} a_{1}\left(F_{1} \backslash A\right)+\cdots+\epsilon_{s} a_{s}\left(F_{s} \backslash A\right)
$$

is a $(d-|A|)$-cycle in $\mathrm{lk}_{\Gamma}(A)$ that is not a boundary in $\mathrm{lk}_{\Gamma}(A)$;

2. $\tilde{H}_{d-|A|}\left(\mathrm{lk}_{\Gamma}(A), k\right) \neq 0$;

3. $A=F_{1} \cap \ldots \cap F_{s}$. 
Proof. The case $|A|=d+1$ will result in $\operatorname{lk}_{\Gamma}(A)=\{\emptyset\}$ which has (-1)-homology. So we can assume that $0 \leqslant|A| \leqslant d$. To prove Statement 1 we will proceed using induction on $a=|A|$. If $a=0$, then $\mathrm{lk}_{\Gamma}(A)=\Gamma, \Sigma_{A}=\Sigma$ and there is nothing to prove.

Suppose $a>0, A=\left\{v_{1}, \ldots, v_{a}\right\}, A^{\prime}=\left\{v_{1}, \ldots, v_{a-1}\right\}$ (or $A^{\prime}=\emptyset$ when $\left.a=1\right)$ and $\Gamma^{\prime}=1 \mathrm{k}_{\Gamma}\left(A^{\prime}\right)$, and suppose without loss of generality

$$
A^{\prime} \subseteq F_{1} \cap \ldots \cap F_{t} \text { and } A^{\prime} \nsubseteq F_{j} \text { for } j>t \geqslant s .
$$

By the induction hypothesis, for some $\epsilon_{i}^{\prime} \in\{ \pm 1\}$ there is a $(d-(a-1))$-cycle

$$
\Sigma_{A^{\prime}}=a_{1} \epsilon_{1}^{\prime}\left(F_{1} \backslash A^{\prime}\right)+\cdots+a_{t} \epsilon_{t}^{\prime}\left(F_{t} \backslash A^{\prime}\right)
$$

in $\Gamma^{\prime}$ that is not a boundary in $\Gamma^{\prime}$ and $\tilde{H}_{d-(a-1)}\left(\Gamma^{\prime}, k\right) \neq 0$. In particular, we must have $t \neq s$ as otherwise the support complex of $\Sigma_{A^{\prime}}$ would be a cone with every facet containing $v_{a}$, a contradiction.

We know that $v_{a} \in\left(F_{i} \backslash A^{\prime}\right)$ if and only if $i \leqslant s$. Depending on the orientation of the faces of the complex $\Gamma^{\prime}$, for some $\epsilon_{i}^{\prime \prime} \in\{ \pm 1\}$, we can write

$$
\begin{aligned}
0 & =\partial\left(\Sigma_{A^{\prime}}\right) \\
& =\epsilon_{1}^{\prime} a_{1} \partial\left(F_{1} \backslash A^{\prime}\right)+\cdots+\epsilon_{t}^{\prime} a_{t} \partial\left(F_{t} \backslash A^{\prime}\right) \\
& =\epsilon_{1}^{\prime \prime} \epsilon_{1}^{\prime} a_{1}\left(F_{1} \backslash A\right)+\cdots+\epsilon_{s}^{\prime \prime} \epsilon_{s}^{\prime} a_{s}\left(F_{s} \backslash A\right)+\mathcal{U}+\partial\left(\epsilon_{s+1}^{\prime} a_{s+1} F_{s+1} \backslash A^{\prime}+\cdots+\epsilon_{t}^{\prime} a_{t} F_{t} \backslash A^{\prime}\right)
\end{aligned}
$$

where $\mathcal{U}$ consists of all the summands above which contain the vertex $v_{a}$, and hence

$$
\mathcal{U}=\sum_{j=1}^{s} \epsilon_{j}^{\prime} a_{j}\left(\partial\left(F_{j} \backslash A^{\prime}\right)-\epsilon_{j}^{\prime \prime} F_{j} \backslash A\right)=0 .
$$

If we set $\epsilon_{i}=\epsilon_{i}^{\prime \prime} \epsilon_{i}^{\prime}$ and $\Sigma_{A}=\epsilon_{1} a_{1}\left(F_{1} \backslash A\right)+\cdots+\epsilon_{s} a_{s}\left(F_{s} \backslash A\right)$ it follows that

$$
\Sigma_{A}=-\partial\left(\epsilon_{s+1}^{\prime} a_{s+1}\left(F_{s+1} \backslash A^{\prime}\right)+\cdots+\epsilon_{t}^{\prime} a_{t}\left(F_{t} \backslash A^{\prime}\right)\right)
$$

and

$$
\partial\left(\Sigma_{A}\right)=-\partial^{2}\left(\epsilon_{s+1}^{\prime} a_{s+1}\left(F_{s+1} \backslash A^{\prime}\right)+\cdots+\epsilon_{t}^{\prime} a_{t}\left(F_{t} \backslash A^{\prime}\right)\right)=0 .
$$

So $\Sigma_{A}$ is a $(d-a)$-cycle in $\mathrm{lk}_{\Gamma^{\prime}}\left(v_{a}\right)=\mathrm{lk}_{\Gamma}(A)$ by Lemma 3.1 (and since $\left.v_{a} \in \Gamma^{\prime}\right)$. Since $\operatorname{dim}\left(\mathrm{lk}_{\Gamma}(A)\right)=$ $d-|A|$, the $(d-|A|)$-cycle $\Sigma_{A}$ is not a boundary in $\mathrm{k}_{\Gamma}(A)$. Therefore, $\tilde{H}_{d-|A|}(\Gamma, k) \neq 0$, proving Statement 2.

To see Statement 3, note that if $F_{1} \ldots, F_{S}$ all contain a vertex outside $A$, then the support complex of $\Sigma_{A}$ would be a cone, contradicting Statement 2.

Example 3.3. Let $\Gamma=\langle x y, z u, z v, u v\rangle$, which is the Alexander dual of the simplicial complex $\Gamma$ in Example 2.4 


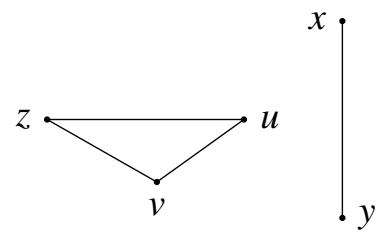

As stated in Theorem 3.2, $\Gamma$ is a 1-dimensional simplicial complex and has $\Sigma=u z+z v+v u$ as a 1-cycle so that $\tilde{H}_{1}(\Gamma, k) \neq 0$. Taking $A=\{z\}$, then $\Sigma_{A}=u-v$ is a 0 -cycle in $\operatorname{lk}_{\Gamma}(A)=\langle u, v\rangle$ with $\tilde{H}_{0}\left(\mathrm{lk}_{\Gamma}(A), k\right) \neq 0$.

Corollary 3.4. Let $k$ be a field, $\Gamma$ a d-dimensional simplicial complex with $\tilde{H}_{d}(\Gamma, k) \neq 0$, and let $\Sigma$ be a d-cycle in $\Gamma$ which is not a boundary. Let $A$ be a face of the support complex of $\Sigma$, and suppose $F_{1} \ldots, F_{q}$ are the facets of $\Gamma$ that contain $A$. Then

$$
A=\bigcap_{j=1}^{q} F_{j}
$$

Proof. Since $\operatorname{lk}_{\Gamma}(A)=\left\langle F_{1} \backslash A, \ldots, F_{q} \backslash A\right\rangle$, if there is a vertex of $\bigcap_{j=1}^{q} F_{j}$ which is not in $A$, then $\mathrm{k}_{\Gamma}(A)$ would be a cone, and would therefore have no homology, contradicting Theorem 3.2 .

Theorem 3.5 below is a formal statement on breaking homological cycles. We refer the reader to parts (2) and (3) of Example 2.4 where we demostrated the theorem's statement. Note also that the case in part (1) of Example 2.4 follows the same pattern, though a proof is not known yet.

Theorem 3.5 (Breaking up cycles on links). Let $k$ be a field and $\Gamma=\left\langle F_{1}, \ldots, F_{r}\right\rangle$ be $a d$ dimensional simplicial complex such that

$$
\tilde{H}_{d}(\Gamma, k) \neq 0 \text { and } d+2=a+b \text { for some } a, b>0 .
$$

Suppose $\Gamma$ contains a d-dimensional cycle

$$
\Sigma=\sum_{j=1}^{q} a_{j} F_{j}
$$

supported on the facets $F_{1}, \ldots, F_{q}$ of $\Gamma$, and $\Sigma$ is not boundary in $\Gamma$. Then there are subsets $A, B \subseteq$ $[q] \subseteq[r]$ with

$$
F=\bigcap_{j \in A} F_{j} \text { and } G=\bigcap_{j \in B} F_{j}
$$

such that

1. $F \cap G=\emptyset$;

2. $F \cup G \notin \Gamma$; 
3. $\tilde{H}_{a-2}\left(\mathrm{lk}_{\Gamma}(F), k\right) \neq 0$ and $\tilde{H}_{b-2}\left(\mathrm{lk}_{\Gamma}(G), k\right) \neq 0$.

Moreover, if $a, b>1, F$ and $G$ and $\epsilon_{j}, \delta_{j} \in\{ \pm 1\}$ could be chosen to additionally satisfy:

4. $|F|=b$ and $|G|=a$;

5. $\Sigma_{F}=\sum_{j \in A} \epsilon_{j} a_{j}\left(F_{j} \backslash F\right)$ is an $(a-2)$-cycle in $\mathrm{lk}_{\Gamma}(F)$ which is not a boundary ;

6. $\Sigma_{G}=\sum_{j \in B} \delta_{j} a_{j}\left(F_{j} \backslash G\right)$ is a $(b-2)$-cycle in $\mathrm{lk}_{\Gamma}(G)$ which is not a boundary.

Proof. Set $i=d+2$. We first consider the case $b=1$ and $a=i-1$. If $a=1$, then $d=0$ and $\Gamma$ is disconnected. Let $F$ and $G$ be two facets each belonging to a distinct connected component of $\Gamma$. Then we clearly have $F \cap G=\emptyset$ and $F \cup G \notin \Gamma$. Moreover, $\operatorname{lk}_{\Gamma}(F)=\mathrm{lk}_{\Gamma}(G)=\{\emptyset\}$ and so

$$
\tilde{H}_{a-2}\left(\mathrm{lk}_{\Gamma}(F), k\right)=\tilde{H}_{b-2}\left(\mathrm{lk}_{\Gamma}(G), k\right)=\tilde{H}_{-1}(\{\emptyset\}, k) \neq 0
$$

as desired.

If $b=1$ and $a=i-1>1$, then $d=a+b-2>0$. By Theorem 3.2, if we take a vertex $v$ in the support complex of $\Sigma$, then $\tilde{H}_{i-3}\left(\mathrm{lk}_{\Gamma}(v), k\right) \neq 0$.

Since $\Sigma$ is a cycle, not all of $F_{1}, \ldots, F_{q}$ contain $v$. Let $G$ be one of the facets $F_{1}, \ldots, F_{q}$ that does not contain $v$. Then $F \cap G=\emptyset$ and $F \cup G \notin \Gamma$ (as $G$ is a facet), and moreover

$$
\tilde{H}_{a-2}\left(\operatorname{lk}_{\Gamma}(F), k\right)=\tilde{H}_{i-3}\left(\operatorname{lk}_{\Gamma}(v), k\right) \neq 0 \text { and } \tilde{H}_{b-2}\left(\operatorname{lk}_{\Gamma}(G), k\right)=\tilde{H}_{-1}(\{, k) \emptyset\} \neq 0 .
$$

Now suppose $a, b \geqslant 2$ and $a=i-b$. Suppose $F_{1}=\left\{w_{1}, v_{1}, \ldots, v_{i-2}\right\}$. Then since $F_{1}$ is in the support of the $(i-2)$-cycle $\Sigma,\left\{w_{1}, v_{2}, \ldots, v_{i-2}\right\}$ must appear in another one of the $F_{j}$ in the support of $\Sigma$, say $F_{2}$. Suppose $F_{2}=\left\{w_{1}, w_{2}, v_{2}, \ldots, v_{i-2}\right\}$. Considering that $a=i-b \leqslant i-2$, let

$$
G=\left\{v_{1}, \ldots, v_{a}\right\} \text { and } F=\left\{v_{a+1}, \ldots, v_{i-2}, w_{1}, w_{2}\right\} .
$$

Then $|G|=a$ and $|F|=i-2+2-a=b$. Moreover $F \cap G=\emptyset$ by construction, and if $i-2=d$, then $F \cup G \notin \Gamma$ since $|F \cup G|=d+2$ which is larger than the size of any face of $\Gamma$.

By Theorem 3.2, and noting that $i-2-|G|=b-2$ and $i-2-|F|=a-2$, we have

$$
\tilde{H}_{a-2}\left(\mathrm{lk}_{\Gamma}(F), k\right) \neq 0 \text { and } \tilde{H}_{b-2}\left(\mathrm{lk}_{\Gamma}(G), k\right) \neq 0,
$$

conditions 5 and 6 are satisfied, and if

$$
A=\left\{j \in[q]: F \subset F_{j}\right\} \text { and } B=\left\{j \in[q]: G \subset F_{j}\right\}
$$

then

$$
F=\bigcap_{j \in A} F_{j} \text { and } G=\bigcap_{j \in B} F_{j}
$$


Another version of Theorem 3.5 below is one which gives lower-dimensional cycles in induced subcomplexes.

Corollary 3.6 (Breaking up cycles). Let $\Gamma$ be a simplicial complex on the vertex set $\left\{x_{1}, \ldots, x_{n}\right\}$, and suppose $\tilde{H}_{d-2}(\Gamma, k) \neq 0$, where $d$ is the smallest possible size of a nonface of $\Gamma$. Suppose $n-d+1=a+b$, where $a$ and $b$ are positive integers. Then there are nonempty subsets $C, D \subseteq$ $\left\{x_{1}, \ldots, x_{n}\right\}$ such that

1. $C \cup D=\left\{x_{1}, \ldots, x_{n}\right\}$;

2. $C \cap D \in \Gamma$;

3. $\tilde{H}_{|C|-a-1}\left(\Gamma_{C}, k\right) \neq 0$ and $\tilde{H}_{|D|-b-1}\left(\Gamma_{D}, k\right) \neq 0$.

Proof. By Alexander duality - see Prop. 5.1.10 and the discussion preceding Prop. 5.1.8 in [18]we have that $\tilde{H}_{n-d-1}\left(\Gamma^{\vee}, k\right) \neq 0$. Now $d$ is the smallest possible size of a nonface of $\Gamma$, so by the definition of Alexander duals, $\operatorname{dim}\left(\Gamma^{\vee}\right)=n-d-1$.

Suppose $\Gamma^{\vee}=\left\langle F_{1}, \ldots, F_{r}\right\rangle$. If $n-d+1=a+b$, then, by Theorem 3.5 , there are subsets $A$ and $B$ of $[r]$ such that

$$
F=\bigcap_{j \in A} F_{j} \text { and } G=\bigcap_{j \in B} F_{j}
$$

and

(i) $F \cap G=\emptyset$;

(ii) $F \cup G \notin \Gamma^{\vee}$;

(iii) $\tilde{H}_{a-2}\left(\mathrm{lk}_{\Gamma^{\vee}}(F), k\right) \neq 0$ and $\tilde{H}_{b-2}\left(\mathrm{lk}_{\Gamma^{\vee}}(G), k\right) \neq 0$.

Now let

$$
C=F^{c}=\bigcup_{j \in A} F_{j}^{c} \text { and } D=G^{c}=\bigcup_{j \in B} F_{j}^{c} .
$$

Then by (i), $C \cup D=(F \cap G)^{c}=\left\{x_{1}, \ldots, x_{n}\right\}$. By (ii), $(C \cap D)^{c}=F \cup G \notin \Gamma^{\vee}$ so $C \cap D \in \Gamma$. Finally by (iii) and Equation (2), $\tilde{H}_{|C|-a-1}\left(\Gamma_{C}, k\right) \neq 0$ and $\tilde{H}_{|D|-b-1}\left(\Gamma_{D}, k\right) \neq 0$.

Theorem 3.7 (Subadditivity of syzygies of square-free monomial ideals). If I is a square-free monomial ideal in the polynomial ring $S=k\left[x_{1}, \ldots, x_{n}\right]$ where $k$ is a field, and $d$ is the smallest possible degree of a generator of $I$. Suppose $i=n-d+1, \beta_{i, n}(S / I) \neq 0$ and $i=a+b$, for some positive integers $a$ and $b$. Then $t_{i} \leqslant t_{a}+t_{b}$.

Proof. By Hochster's formula (Equation (2)), if $\Gamma=\mathcal{N}(I)$, then

$$
\beta_{n-d+1, n}(S / I)=\beta_{n-d+1, x_{1} \cdots x_{n}}(S / I)=\operatorname{dim}_{k} \tilde{H}_{d-2}(\Gamma, k) \neq 0 .
$$

If $n-d+1=a+b$, then by Corollary 3.6 , there are nonempty subsets $C, D \subseteq\left\{x_{1}, \ldots, x_{n}\right\}$ such that

$$
C \cup D=\left\{x_{1}, \ldots, x_{n}\right\} \text { and } C \cap D \in \Gamma \text {, }
$$


and

$$
\tilde{H}_{|C|-a-1}\left(\Gamma_{C}, k\right) \neq 0 \text { and } \tilde{H}_{|D|-b-1}\left(\Gamma_{D}, k\right) \neq 0 .
$$

By Equation (2), this means that

$$
\beta_{a,|C|}(S / I) \neq 0 \text { and } \beta_{b,|D|}(S / I) \neq 0,
$$

so that $t_{a} \geqslant|C|$ and $t_{b} \geqslant|D|$. Putting this all together we get

$$
t_{a}+t_{b} \geqslant|C|+|D| \geqslant n=t_{i}
$$

which settles our claim.

Discussion 3.8. Given a square-free monomial ideal $I$ if we are looking for top degree Betti numbers, by Hochster's formula (Equation (2))

$$
\beta_{n-i-1, n}(S / I)=\operatorname{dim}_{k} \tilde{H}_{i}(\Gamma, k) .
$$

Now if $d$ is the smallest possible degree of a generator of $I$, then all monomials of degree $\leqslant d-1$ are not in $I$, which means all possible faces of dimension $\leqslant d-2$ are in $\Gamma=\mathcal{N}(I)$. This means that the smallest index $i$ with $\tilde{H}_{i}(\Gamma, k) \neq 0$ is $d-2$, that is

$$
\tilde{H}_{i}(\Gamma, k)=0 \text { for } i<d-2
$$

and hence

$$
\beta_{j, n}(S / I)=0 \text { for } j=n-i-1>n-d+1 .
$$

So $n-d+1$ is the maximum homological degree where we could have a nonvanishing top degree Betti number. We do not have an example of our setting where $n-d+1$ is not the projective dimension. After comparing with bounds on the projective dimension of $S / I$ given by Dao and Schweig [8, Theorem 3.2, Remark 3.4] in terms of dominance parameters of clutters, we concluded that $n-d+1$ is often either the projective dimension of $S / I$ or very close to it, though we were not able to determine how close.

Example 3.9. Let $I=(x y z, x z v, x u v, y z u, y u v)$ be an ideal of $S=k[x, y, z, u, v]$ in 5 variables. Here the smallest degree of a generator of $I$ is $d=3$, so $n-d+1=3$, so we pick $a=1$ and $b=2$. According to Macaulay2 [23] the Betti table of $S / I$ is

$\begin{array}{rllll} & 0 & 1 & 2 & 3 \\ \operatorname{total}: & 1 & 5 & 5 & 1 \\ 0: & 1 & . & . & . \\ 1: & . & . & . & . \\ 2: & . & 5 & 5 & 1\end{array}$

which verifies that

$$
t_{3}=5, t_{2}=4, t_{1}=3 \Longrightarrow t_{3}<t_{1}+t_{2}=7 \text {. }
$$

Example 3.10. In Example 2.4 $I=(x z, y z, x u, y u, x v, y v, z u v)$ is a square-free monomial ideal in 5 variables where $d=2$ and $n-d+1=4$. According to the Betti table of $I, t_{4}=5, t_{3}=5, t_{2}=4$ and $t_{1}=3$. Here $t_{4}<t_{1}+t_{3}=8$ and $t_{4}<2 t_{2}=8$. Note that we also have $\beta_{3,5}(S / I) \neq 0$ where $3<4=n-d+1$ while still we have $t_{3}<t_{1}+t_{2}=7$. 


\section{Special cases of breaking up simplicial homology}

In this section, we consider breaking up special classes of cycles, where we can provide a combinatorial description for the lower-dimensional cycles.

\subsection{The case of a disconnected simplicial complex}

We begin with an example.

Example 4.1. Let $\mathcal{N}(I)=\Gamma=\langle u v, x y, y z, x z\rangle$ be a simplicial complex on $n=5$ vertices.

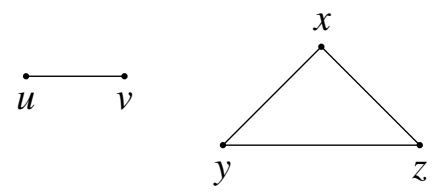

Here $\tilde{H}_{0}(\Gamma, k) \neq 0$ and hence $\beta_{4, u v x y z}(S / I) \neq 0$. If $4=a+b$, then using Corollary 3.6 we have the following two cases to consider.

1. $a=1$ and $b=3$. Let $C=\{u, x\}$ and $D=\{u, v, y, z\}$. Then $C \cup D=\{u, v, x, y, z\}, C \cap D=\{u\} \in \Gamma$ and

$$
\tilde{H}_{|C|-a-1}\left(\Gamma_{C}, k\right)=\tilde{H}_{0}(\langle u, x\rangle, k) \neq 0 \text { and } \tilde{H}_{|D|-b-1}\left(\Gamma_{D}, k\right)=\tilde{H}_{0}(\langle u v, y z\rangle, k) \neq 0 \text {. }
$$

2. $a=b=2$. Let $C=\{u, x, v\}$ and $D=\{u, y, z\}$. Then $C \cup D=\{u, v, x, y, z\}, C \cap D=\{u\} \in \Gamma$ and

$$
\tilde{H}_{|C|-a-1}\left(\Gamma_{C}, k\right)=\tilde{H}_{0}(\langle u v, x\rangle, k) \neq 0 \text { and } \tilde{H}_{|D|-b-1}\left(\Gamma_{D}, k\right)=\tilde{H}_{0}(\langle u, y z\rangle, k) \neq 0 .
$$

In general if $\Gamma$ is a disconnected complex on $n$ vertices with Stanley-Reisner ideal $I$, then $\beta_{n-1, n}(S / I) \neq 0$, and if $n-1=a+b$ for some $a, b>0$, then we can always find disconnected induced subcomplexes $\Gamma_{C}$ and $\Gamma_{D}$ where $C=a+1$ and $D=b+1$, as in the example above. Below we demonstrate how this can be done.

If $\Gamma$ is disconnected, then it has the form

$$
\Gamma=\Gamma_{1} \cup \cdots \cup \Gamma_{t}
$$

where $\Gamma_{1}, \ldots, \Gamma_{t}$ are connected components and $t>1$. In this case, $\left|V\left(\Gamma_{i}\right)\right| \geqslant 1$ for all $1 \leqslant i \leqslant t$, $V(\Gamma)=V\left(\Gamma_{1}\right) \cup \cdots \cup V\left(\Gamma_{t}\right)$ and $V\left(\Gamma_{k}\right) \cap V\left(\Gamma_{l}\right)=\emptyset$ for all $1 \leqslant k<l \leqslant t$.

Without loss of generality and up to renaming the variables, we can assume the following:

- $\left|V\left(\Gamma_{1}\right)\right| \leqslant\left|V\left(\Gamma_{2}\right)\right| \leqslant \cdots \leqslant\left|V\left(\Gamma_{t}\right)\right|$,

- $x_{k} \in V\left(\Gamma_{k}\right)$ for $1 \leqslant k \leqslant t$,

- $V\left(\Gamma_{1}\right)=\left\{x_{1}, x_{t+1}, \ldots, x_{t+\left|V\left(\Gamma_{1}\right)\right|-1}\right\}$ 
- $V\left(\Gamma_{k}\right)=\left\{x_{k}, x_{\left(t+\left|V\left(\Gamma_{1}\right)\right|+\cdots+\left|V\left(\Gamma_{k-1}\right)\right|-k+2\right)}, \ldots, x_{\left(t+\left|V\left(\Gamma_{1}\right)\right|+\cdots+\left|V\left(\Gamma_{k}\right)\right|-k\right)}\right\}$ for each $1<k \leqslant t$.

Example 4.2. The simplicial complex $\Gamma$ in Example 4.1 can be relabeled and written as $\Gamma=\Gamma_{1} \cup \Gamma_{2}$ where $\Gamma_{1}=\left\langle x_{1} x_{3}\right\rangle$ and $\Gamma_{2}=\left\langle x_{2} x_{4}, x_{4} x_{5}, x_{2} x_{5}\right\rangle$.

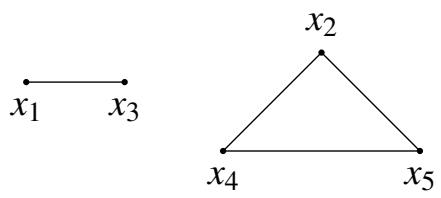

For each $1 \leqslant a<n-1$, define

$$
C=\left\{x_{1}, x_{2}, \ldots, x_{a+1}\right\} \text { and } D=\left\{x_{1}, x_{a+2}, \ldots, x_{n}\right\} .
$$

Clearly $C \cup D=\left\{x_{1}, \ldots, x_{n}\right\},|C|=a+1,|D|=n-a$ and $C \cap D=\left\{x_{1}\right\} \in \Gamma$. Moreover, it is easy to see that both $\Gamma_{C}$ and $\Gamma_{D}$ are disconnected induced subcomplexes of $\Gamma$ on the subsets $\left\{x_{1}, x_{2}, \ldots, x_{a+1}\right\}$ and $\left\{x_{1}, x_{a+2}, \ldots, x_{n}\right\}$, respectively. Therefore, if $b=n-a-1$

$$
\tilde{H}_{|C|-a-1}\left(\Gamma_{C}, k\right)=\tilde{H}_{0}\left(\Gamma_{C}, k\right) \neq 0 \text { and } \tilde{H}_{|D|-b-1}\left(\Gamma_{D}, k\right)=\tilde{H}_{0}\left(\Gamma_{D}, k\right) \neq 0 .
$$

\subsection{The case of a graph cycle}

Recall that a cycle in a graph $G$ is an ordered list of distinct vertices $x_{1}, \ldots, x_{n}$ where the edges are $x_{i-1} x_{i}$ for $2 \leqslant i \leqslant n$ and $x_{n} x_{1}$. Graph cycles characterize nontrivial 1-homology in simplicial complexes; see for example Theorem 3.2 in [7].

Suppose $\Gamma$ is a simplicial complex on the set $\left\{x_{1}, \ldots, x_{n}\right\}$ that is the support complex of a faceminimal graph cycle, so that $\tilde{H}_{1}(\Gamma, k) \neq 0$. This means that $\beta_{n-2, n}(S / I) \neq 0$. Suppose $n-2=a+b$ for some $a, b>0$.

Without loss of generality, $\Gamma$ can be written in the form

$$
\Gamma=\left\langle x_{1} x_{2}, x_{2} x_{3}, \ldots, x_{n-1} x_{n}, x_{n} x_{1}\right\rangle .
$$

For $1 \leqslant a<n-2$, define

$$
C=\left\{x_{1}, x_{3}, x_{4}, \ldots, x_{a+2}\right\} \text { and } D=\left\{x_{2}, x_{a+3}, \ldots, x_{n}\right\} .
$$

Clearly, $C \cup D=\left\{x_{1}, \ldots, x_{n}\right\},|C|=a+1,|D|=n-a-1$ and $C \cap D=\emptyset \in \Gamma$. Moreover, it is easy to see that both $\Gamma_{C}$ and $\Gamma_{D}$ are disconnected induced subcomplexes of $\Gamma$ on the subsets $\left\{x_{1}, x_{3}, x_{4}, \ldots, x_{a+2}\right\}$ and $\left\{x_{2}, x_{a+3}, \ldots, x_{n}\right\}$, respectively. Therefore,

$$
\tilde{H}_{|C|-a-1}\left(\Gamma_{C}, k\right)=\tilde{H}_{0}\left(\Gamma_{C}, k\right) \neq 0
$$

and

$$
\tilde{H}_{|D|-b-1}\left(\Gamma_{D}, k\right)=\tilde{H}_{0}\left(\Gamma_{D}, k\right) \neq 0
$$

where $b=n-a-2$. 
Example 4.3. Let $\mathcal{N}(I)=\Gamma=\left\langle x_{1} x_{2}, x_{2} x_{3}, x_{3} x_{4}, x_{4} x_{5}, x_{1} x_{5}\right\rangle$.

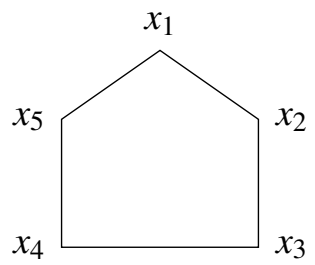

Then $\tilde{H}_{1}(\Gamma, k) \neq 0$ and hence $\beta_{3, x_{1} \cdots x_{5}}(S / I) \neq 0$. Taking $a=1$ and $b=2$, set $C=\left\{x_{1}, x_{3}\right\}$ and $D=\left\{x_{2}, x_{4}, x_{5}\right\}$. Then

$$
\tilde{H}_{|C|-a-1}\left(\Gamma_{C}, k\right)=\tilde{H}_{0}\left(\left\langle x_{1}, x_{3}\right\rangle, k\right) \neq 0 \text { and } \tilde{H}_{|D|-b-1}\left(\Gamma_{D}, k\right)=\tilde{H}_{0}\left(\left\langle x_{2}, x_{4} x_{5}\right\rangle, k\right) \neq 0 .
$$

\section{The case of general monomial ideals}

The polarization [16] of a monomial ideal $I$ is a method to transform $I$ to a square-free monomial ideal, by adding new variables to the polynomial ring. The procedure is described below.

Definition 5.1 (Polarization). Let $I$ be minimally generated by monomials $\mathbf{m}_{1}, \ldots \mathbf{m}_{q}$ in the polynomial ring $R=k\left[x_{1}, \ldots, x_{n}\right]$. For $i \in\{1, \ldots, n\}$, let

$$
p_{i}= \begin{cases}1 & \text { if } x_{i} \nmid \mathbf{m}_{u} \text { for every } u \in[q] \\ \max \left\{j: x_{i}^{j} \mid \mathbf{m}_{u} \text { for some } u \in[q]\right\} & \text { otherwise. }\end{cases}
$$

Let $S$ be the polynomial ring in $\mathbf{p}=p_{1}+\cdots+p_{n}$ variables

$$
S=k\left[x_{i, j}: 1 \leqslant i \leqslant n, 1 \leqslant j \leqslant p_{i}\right]
$$

and let the polarization of $I$ be the square-free monomial ideal

$$
\mathcal{P}(I)=\left(\mathcal{P}\left(\mathbf{m}_{1}\right), \ldots, \mathcal{P}\left(\mathbf{m}_{q}\right)\right)
$$

where, if $\mathbf{m}=x_{a_{1}}{ }^{b_{1}} \cdots x_{a_{c}}{ }^{b_{c}}$ where the $a_{i}$ are distinct integers in $\{1, \ldots, n\}$ and $1 \leqslant b_{i} \leqslant p_{i}$ for $1 \leqslant i \leqslant c$, then

$$
\mathcal{P}(\mathbf{m})=x_{a_{1}, 1} \cdots x_{a_{1}, b_{1}} x_{a_{2}, 1} \cdots x_{a_{2}, b_{2}} \cdots x_{a_{c}, 1} \cdots x_{a_{c}, b_{c}} .
$$

Example 5.2. If $I=\left(x^{2}, x y^{3} z^{2}\right) \subseteq k[x, y, z]$ then its polarization is the square-free monomial ideal $\mathcal{P}(I)=\left(x_{1} x_{2}, x_{1} y_{1} y_{2} y_{3} z_{1} z_{2}\right)$ in the polynomial ring $k\left[x_{1}, x_{2}, y_{1}, y_{2}, y_{3}, z_{1}, z_{2}\right]$.

Corollary 5.3 (Subadditivity of syzygies of monomial ideals). If I is a monomial ideal in the polynomial ring $R=k\left[x_{1}, \ldots, x_{n}\right]$ where $k$ is a field, $d$ is the smallest possible degree of a generator of $I$, and $\mathbf{p}$ is defined as in Definition 5.1 Suppose $i=\mathbf{p}-d+1, \beta_{i, \mathbf{p}}(R / I) \neq 0$ and $i=a+b$, for some positive integers $a$ and $b$. Then $t_{i} \leqslant t_{a}+t_{b}$. 
Proof. Let $I=\left(\mathbf{m}_{1}, \ldots, \mathbf{m}_{q}\right)$, whose polarization is the square-free monomial ideal $\mathcal{P}(I)$ in the polynomial ring $S$ in $\mathbf{p}$ variables in Definition 5.1. Since $\beta_{i, \mathbf{p}}(R / I) \neq 0$, we must have $\beta_{i, \mathbf{m}}(R / I) \neq 0$ for some $\mathbf{m} \in \operatorname{LCM}(I)$. On the other hand, $\mathbf{p}$ is the largest possible degree for a monomial in $\operatorname{LCM}(I)$, and so $\mathbf{m}=\operatorname{lcm}\left(\mathbf{m}_{1}, \ldots, \mathbf{m}_{q}\right)$, the top monomial in the lcm lattice of $I$.

Now the two $1 \mathrm{~cm}$ lattices $\operatorname{LCM}(I)$ and $\operatorname{LCM}(\mathcal{P}(I))$ are isomorphic $([17])$, and the degree $\mathbf{p}$ square-free monomial $\mathcal{P}(\mathbf{m})$ sits on top of the lattice $\operatorname{LCM}(\mathcal{P}(I))$, and so $\beta_{i, \mathbf{p}}(S / \mathcal{P}(I)) \neq 0$. Now since $\operatorname{deg}\left(\mathbf{m}_{i}\right)=\operatorname{deg}\left(\mathcal{P}\left(\mathbf{m}_{i}\right)\right)$ for all $1 \leqslant i \leqslant q$, the conditions for Theorem 3.7 hold, and therefore $t_{i} \leqslant t_{a}+t_{b}$ holds for the ideal $\mathcal{P}(I)$. But as the graded Betti numbers of $I$ and $\mathcal{P}(I)$ are equal, the inequality also holds for $I$, and we are done.

Example 5.4. Let $I=\left(x y^{2}, x y z, y^{3}, y^{2} z\right)$ be an ideal of $R=k[x, y, z]$. Here $\mathbf{p}=5$ and the smallest degree of a generator is $d=3$, so $\mathbf{p}-d+1=3$. We pick $a=1$ and $b=2$. According to Macaulay2 [23] the Betti table of $R / I$ is

$\begin{array}{rllll} & 0 & 1 & 2 & 3 \\ \text { total : } & 1 & 4 & 4 & 1 \\ 0: & 1 & . & . & . \\ 1: & . & . & . & . \\ 2: & . & 4 & 4 & 1\end{array}$

which verifies that

$$
t_{3}=5, t_{2}=4, t_{1}=3 \Longrightarrow t_{3}<t_{1}+t_{2}=7
$$

\section{Final Remarks}

Question 2.1, Question 2.2, Question 2.3 and Question 2.5 are all equivalent, though their different settings allow the application of different (inductive) tools. All of them are open in their full generality as far as we know, though each can be answered positively for certain classes of ideals or combinatorial objects. A positive answer to either would settle the subadditivity question for monomial ideals in a polynomial ring.

\section{References}

[1] A. Abedelfatah, Some results on the subadditivity condition of syzygies, arXiv:2001.01136v1 [math.AC] (2020).

[2] L. Avramov, A. Conca and S. Iyengar, Subadditivity of syzygies of Koszul algebras, Math. Ann. 361, no. 1-2, $511-534$ (2015).

[3] A. Abedelfatah and E. Nevo, On vanishing patterns in j-strands of edge ideals, J. Algebraic Combin. 46, no. 2, 287-295 (2017).

[4] K. Baclawski, Galois connections and the Leray spectral sequence, Advances in Math. 25, no. 3, $191-215$ (1977). 
[5] M. Bigdeli and J. Herzog, Betti diagrams with special shape, Homological and Computational Methods in Commutative Algebra, 33 - 52 (2017).

[6] W. Bruns, J. Herzog, Cohen-Macaulay Rings, Cambridge University Press, Cambridge, Revised Edition, 1997.

[7] E. Connon, On d-dimensional cycles and the vanishing of simplicial homology, arXiv:1211.7087 [math.AC] (2013).

[8] H. Dao and J. Schweig, Bounding the projective dimension of a squarefree monomial ideal via domination in clutters, Proceedings of the American Mathematical Society, Volume 143, Number 2. 555 - 565 (2015).

[9] N. Erey and S. Faridi, Multigraded Betti numbers of simplicial forests, J. Pure Appl. Algebra $218,1800-1805$ (2014).

[10] N. Erey and S. Faridi, Betti numbers of monomial ideals via facet covers, J. Pure Appl. Algebra 220, no. 5, 1990 - 2000 (2016).

[11] D. Eisenbud, C. Huneke, and B. Ulrich, The regularity of Tor and graded Betti numbers, Amer. J. Math. 128, no. 3, 573 - 605 (2006).

[12] S. El Khoury and H. Srinivasan, A note on the subadditivity of Syzygies, Journal of Algebra and its Applications, vol.16, no.9, 1750177 (2017).

[13] S. Faridi, Lattice complements and the subadditivity of syzygies of simplicial forests, Journal of Commutative Algebra, Volume 11, Number 4, 535 - 546 (2019).

[14] S. Faridi, Simplicial trees are sequentially Cohen-Macaulay, J. Pure Appl. Algebra 190, no. $1-3,121-136$ (2004).

[15] O. Fernández-Ramos and P. Gimenez, Regularity 3 in edge ideals associated to bipartite graphs, J. Algebraic Combin. 39 (2014).

[16] R. Fröberg, On Stanley-Reisner rings, in: Topics in algebra, Banach Center Publications, 26 Part 2, 57 - 70 (1990).

[17] V. Gasharov, I. Peeva, V. Welker, The lcm-lattice in monomial resolutions, Mathematical Research Letters 6, 521 - 532 (1999).

[18] J. Herzog and T. Hibi Monomial Ideals, Graduate Texts in Mathematics, vol. 260, SpringerVerlag, London (2011). http://dx.doi.org/10.1007/978-0-85729-106-6

[19] J. Herzog and H. Srinivasan, On the subadditivity problem for maximal shifts in free resolutions, Commutative Algebra and Noncommutative Algebraic Geometry, II MSRI Publications Volume 68, (2015). 
[20] A. V. Jayanthan and A. Kumar, Subadditivity, strand connectivity and multigradedbetti numbers of monomial ideals, arXiv:2007.15319 (2020).

[21] J. McCullough, A polynomial bound on the regularity of an ideal in terms of half of the syzygies, Math. Res. Letters 19, 555-565 (2012).

[22] J. McCullough, Subadditivity of syzygies of ideals and related problems, preprint, available at https://faculty.sites.iastate.edu/jmccullo/files/inline-files/Subadditivity\%20Survey.pdf.

[23] D. Grayson and M. Stillman, Macaulay2, a software system for research in algebraic geometry. Available at http://www.math.uiuc.edu/Macaulay2/.

[24] I. Peeva, Graded syzygies, Algebra and Applications, 14. Springer-Verlag London, Ltd., London (2011).

[25] V. Reiner and V. Welker, Linear syzygies of Stanley-Reisner ideals, Mathematica Scandinavica, 89(1), 117 - 132 (2001).

[26] D. Taylor, Ideals generated by monomials in an R-sequence, Thesis, University of Chicago (1966). 